Journal of Nepal Mathematical Society (JNMS), Vol. 3, Issue 2 (2020); L. de O. Miranda, L.B.B. Miranda

\title{
Generalization of Dürer's Magic Square and New Methods for Doubly Even Magic Squares
}

\author{
Lohans de Oliveira Miranda ${ }^{1}$, Lossian Barbosa Bacelar Miranda ${ }^{2}$ \\ ${ }^{1}$ Unisul University, Brazil \\ 2 IFPI, Brazil \\ Correspondence to: Lossian Barbosa Bacelar Miranda, Email: lossianm@gmail.com
}

\begin{abstract}
We have established new general methods of building of doubly even magic squares and from these methods we naturally obtain Dürer's magic square. This allows us to define Dürer's magic squares for orders greater than four.
\end{abstract}

Keywords: Arithmetic progressions, Doubly even magic squares, D“urer's magic square, Parity DOI: https://doi.org/10.3126/jnms.v3i2.33955

\section{Introduction}

Jacques Sesiano's studies report that the general methods of constructing doubly even magic squares were made by eastern mathematicians before the eleventh century ([2], pp. 44-88). Here we present new general methods which builds, for each order, new types of magic squares hitherto unknown. These new magic squares have many symmetries, which are amazing. We use the definitions and notations of [1]. A magic square of order $n$ is a square matrix formed by the numbers $1,2,3, \ldots, n^{2}$ and such that the sum of the numbers of each row, each column and each of the two diagonals is equal to $c_{n}=\frac{n^{3}+n}{2}$. We call $c_{n}$ of magic constant ${ }^{1}$ If $n=4 k, k$ positive natural number, the magic square is of type doubly even magic square.

\section{Main Results}

Proposition 2.1. Let $n=4 k, k \in \mathbb{N}^{*}$ and $i, j \in\left\{4 t, t \in I_{\frac{n}{4}}\right\}$. Consider the square matrix

$$
D_{n}=\left(d_{u, v}\right)_{u, v \in I_{n}}=\left(\begin{array}{ccc}
D_{4,4} & \cdots & D_{4, n} \\
\vdots & \ddots & \vdots \\
D_{n, 4} & \cdots & D n, n
\end{array}\right)
$$

of order $n$ determined by blocks of order 4 given by

$$
D_{i, j}=\left(\begin{array}{cccc}
(i-3) n-(j-4) & (i-3) n+(j-2) & (n-i+2) n+(j-1) & (n+4-j) n-(j-1) \\
(n+4-i) n-(j-3) & (n-i+2) n+(j-3) & (i-3) n+j & (i-3) n-(j-2) \\
(i-1) n+(j-2) & (i-1) n-(j-4) & (n+2-i) n-(j-1) & (n-i) n+(j-1) \\
(n-i) n+(j-3) & (n+2-i) n-(j-3) & (i-1) n-(j-2) & (i-1) n+j
\end{array}\right)
$$

Then: i) If $n>4, D_{i, j}$ is a non-normal magic square with a total equal to $2 n^{2}+2$ for each pair $i, j \in$ $\left\{4 t, t \in I_{\frac{n}{4}}\right\}$. If $n=4, D_{i, j}$ is magic square; ii) $D_{n}$ above is a magic square.

Proof.

\footnotetext{
${ }^{1}$ The magic square is non-normal when the sum of the numbers in lines, columns and diagonals are all the same, however, not equal to $c_{n}=\frac{n^{3}+n}{2}$ or the set of numbers that form it is not $I_{n}=\{1,2,3, \ldots, n\}$. In generic cases we sometimes do not know whether the magic square is normal or non-normal. In these cases we will use the word non-normal or we will say that it is not necessarily normal. It would be better to call everyone magic squares and reserve the words normal and non-normal for the two disjunct species. We will not run over the historical evolution of this confusing nomenclature
} 
i) We have,

$(i-3) n-(j-4)+(i-3) n+(j-2)+(n-i+2) n+(j-1)+(n+4-j) n-(j-1)=2 n^{2}+2$.

It is the same with the other three lines. We also have $(i-3) n-(j-4)+(n+4-i) n-(j-3)+(i-$ $1) n+(j-2)+(n-i) n+(j-3)=2 n^{2}+2$. It is the same with the other three columns. Similarly $(i-3) n-(j-4)+(n-i+2) n+(j-3)+(n+2-i) n-(j-1)+(i-1) n+j=2 n^{2}+2$ and $(n-i) n+(j-3)+(i-1) n-(j-4)+(i-3) n+j+(n+4-j) n-(j-1)=2 n^{2}+2$. Therefore, $D_{i, j}$ is a non-normal magic square if $n \neq 4$ and normal if $n=4$.

ii) The sum of the numbers of any line of $D_{n}$ is equal to the sum of the numbers of $n / 4$ matrix lines of type $D_{i, j}$ with $j$ ranging from 4 to $n$ traversing multiples of 4 . Now, these $n / 4$ sums are all equal to $2 n^{2}+2$. Therefore, the sum of the numbers of any line of $D_{n}$ is equal to $(n / 4)\left(2 n^{2}+2\right)=\left(n^{3}+n\right) / 2=c_{n}$, the magic constant. Analogous result is checked for any column. In the case of diagonals, the sum of the elements of the main diagonal of $D_{n}$ is equal to the sum of the sums of the main diagonals of $D_{4,4}, D_{8,8}, D_{12,12}, \ldots, D_{n, n}$. However, each of these has sum of diagonal numbers equal to $2 n^{2}+2$. Therefore, the sum of the elements of the main diagonal of $D_{n}$ is $(n / 4)\left(2 n^{2}+2\right)=c_{n}$. Similarly, we can prove that the sum of the elements of the secondary diagonal of $D_{n}$ is also equal to the magic constant $c_{n}$.

Proposition 2.2. If in the magic square $D_{n}$ we replace $\left(d_{i, 1}, d_{i, 2}, d_{i, 3}, \ldots, d_{i, n}\right)$ by $\left(d_{i, 1}, d_{i+1,2}, d_{i+2,3}\right.$ $\left., \ldots, d_{n, n+1-i}, d_{1, n+2-i}, d_{2, n+3-i}, d_{3, n+4-i}, \ldots, d_{i-1, n}\right), \forall i \in I_{n}, i$ odd number and $\left(d_{k, 1}, d_{k, 2}, d_{k, 3}\right.$ $\left., \ldots, d_{k, n}\right)$ by $\left(d_{k, 1}, d_{k-1,2}, d_{k-2,3}, \ldots, d_{1, k} ; d_{n, k+1}, d_{n-1, k+2}, d_{n-2, k+3}, \ldots, d_{k+1, n}\right), \forall k \in I_{n}, k$ even number then the resulting square matrix $D u_{n}$ will be a semi-magic square. In addition, $D u_{n}$ will be a magic square if and only if $n$ is not a multiple of 8 .

Proof. The first line of $D u_{n}$ is equal to the main diagonal of $D_{n}$ and the line of $n$ order of $D u_{n}$ is equal to the secondary diagonal of $D_{n}$. The columns of $D u_{n}$ have, respectively, the same numbers as the columns of $D_{n}$, except for permutation of its elements. Therefore, the sum of these elements is equal to the magic constant of order $n$. The above established is true for any $n$ multiple of 4 . The rest of the proof follows by direct inspection on each row, column and diagonals, adding the elements of the broken diagonals (alternatively down and up) of $D_{n}$ which start in the first column in the values $(i-3) n+3,(n+4-i) n+2,(n-i) n-1$, $(n-i) n-2, i=1,2,3, \ldots, n$.

\section{Examples}

$$
D_{4}=\left(\begin{array}{cccc}
4 & 6 & 11 & 13 \\
15 & 9 & 8 & 2 \\
14 & 12 & 5 & 3 \\
1 & 7 & 10 & 16
\end{array}\right), \quad D u_{4}=\left(\begin{array}{cccc}
4 & 9 & 5 & 16 \\
15 & 6 & 10 & 3 \\
14 & 7 & 11 & 2 \\
1 & 12 & 8 & 13
\end{array}\right)
$$

Note that $D u_{4}$ is the Dürer's magic square and this fact allows us to establish the following definition.

Definition 3.1. We call Du $u_{4(2 m+1)}$ of generalized Dürer's magic square of order 4(2m+1).

$$
D_{8}=\left(\begin{array}{cccccccc}
8 & 10 & 51 & 61 & 4 & 14 & 55 & 57 \\
63 & 49 & 12 & 6 & 59 & 53 & 16 & 2 \\
26 & 24 & 45 & 35 & 30 & 20 & 41 & 39 \\
33 & 47 & 22 & 28 & 37 & 43 & 18 & 32 \\
40 & 42 & 19 & 29 & 36 & 46 & 23 & 25 \\
31 & 17 & 44 & 38 & 27 & 21 & 48 & 34 \\
58 & 56 & 13 & 3 & 62 & 52 & 9 & 7 \\
1 & 15 & 54 & 60 & 5 & 11 & 50 & 64
\end{array}\right), \quad D u_{8}=\left(\begin{array}{cccccccc}
8 & 49 & 45 & 28 & 36 & 21 & 9 & 64 \\
63 & 10 & 54 & 3 & 27 & 46 & 18 & 39 \\
26 & 47 & 19 & 38 & 62 & 11 & 55 & 2 \\
33 & 24 & 12 & 61 & 5 & 52 & 48 & 25 \\
40 & 17 & 13 & 60 & 4 & 53 & 41 & 32 \\
31 & 42 & 22 & 35 & 59 & 14 & 50 & 7 \\
58 & 15 & 51 & 6 & 30 & 43 & 23 & 34 \\
1 & 56 & 44 & 29 & 37 & 20 & 16 & 57
\end{array}\right) .
$$

The magic constant of order 8 is 260 and the sum of the diagonal numbers is only 196 (note that 260-196= $\left.8^{2}\right)$. 
Journal of Nepal Mathematical Society (JNMS), Vol. 3, Issue 2 (2020); L. de O. Miranda, L.B.B. Miranda

\section{Discussion}

For each magic square produced in accordance with article [3], there is another produced by Proposition 1 above, in a situation of full duality. In [3] odd numbers are placed in ascending order from top to bottom, and in Proposition 1, the same numbers are placed in ascending order from bottom to top. In [3] we established ten properties for the magical squares built there. We expect similar results, too, in the two methods presented here.

\section{References}

[1] Andrews, W. S., 1908, Magic Squares and Cubes, Open Court, Chicago. Available on https://archive.org/details/magicsquarescube00andrrich/page/n6.

[2] Jacques, S., 2019, Magic Squares: Their History and Construction from Ancient Times to AD 1600. Springer Nature Switzerland AG 2019. Available on https://doi.org/10.1007/978-3-030-17993-9.

[3] Miranda, L. de O. and Miranda, L. B. B., 2020, Lohan's Magic Squares and the Gaussian Elimination Method. Journal of Nepal Mathematical Society (JNMS), 3(1), 31-36. 